\title{
Extremal Graphs for a Graph Packing Theorem of Sauer and Spencer
}

\author{
HEMANSHU KAUL and ALEXANDR KOSTOCHKA ${ }^{\dagger}$ \\ Department of Mathematics, University of Illinois at Urbana-Champaign, Urbana, IL 61801, USA \\ (e-mail: hkaul@math.uiuc.edu, kostochk@math.uiuc.edu)
}

Received 30 March 2005; revised 24 August 2005

\begin{abstract}
Let $G_{1}$ and $G_{2}$ be graphs of order $n$ with maximum degree $\Delta_{1}$ and $\Delta_{2}$, respectively. $G_{1}$ and $G_{2}$ are said to pack if there exist injective mappings of the vertex sets into [n], such that the images of the edge sets do not intersect. Sauer and Spencer showed that if $\Delta_{1} \Delta_{2}<\frac{n}{2}$, then $G_{1}$ and $G_{2}$ pack. We extend this result by showing that if $\Delta_{1} \Delta_{2} \leq \frac{n}{2}$, then $G_{1}$ and $G_{2}$ do not pack if and only if one of $G_{1}$ or $G_{2}$ is a perfect matching and the other either is $K_{\frac{n}{2}, \frac{n}{2}}$ with $\frac{n}{2}$ odd or contains $K_{\frac{n}{2}+1}$.
\end{abstract}

\section{Introduction}

Let $G_{1}=\left(V_{1}, E_{1}\right)$ and $G_{2}=\left(V_{2}, E_{2}\right)$ be $n$-vertex graphs with maximum degrees $\Delta\left(G_{i}\right)=\Delta_{i}$ for $i=1,2 . G_{1}$ and $G_{2}$ are said to pack if there exist injective mappings of their vertex sets into $[n]$, such that the images of the edge sets do not intersect. In other words, $G_{1}$ is isomorphic to a subgraph of the complement of $G_{2}$. The study of packings of graphs was started in the 1970s by Bollobás and Eldridge [1, 2], Sauer and Spencer [4], and Catlin [3]. (See the surveys by Wozniak [5] and Yap [6] for later developments in this field.) In particular, Sauer and Spencer [4] proved the following result.

Theorem 1.1 (Sauer and Spencer). Let $G_{1}=\left(V_{1}, E_{1}\right)$ and $G_{2}=\left(V_{2}, E_{2}\right)$ be n-vertex graphs with maximum degrees $\Delta\left(G_{i}\right)=\Delta_{i}$ for $i=1$, 2. If $2 \Delta_{1} \Delta_{2}<n$, then $G_{1}$ and $G_{2}$ pack.

This is sharp for even $n$ : take $G_{1}$ to be a perfect matching on $n$ vertices and $G_{2}$ to be either $K_{\frac{n}{2}, \frac{n}{2}}$ with $\frac{n}{2}$ odd, or containing $K_{\frac{n}{2}+1}$. We are interested in describing the pairs of graphs with $2 \Delta_{1} \Delta_{2}=n$ that do not pack. We show that these examples are the only possibilities, thus somewhat extending the Sauer-Spencer theorem.

\footnotetext{
$\dagger$ Research of this author was partially supported by the NSF grant DMS-0400498 and grant 03-01-00796 of the Russian Foundation for Basic Research.
} 
Theorem 1.2. Let $G_{1}=\left(V_{1}, E_{1}\right)$ and $G_{2}=\left(V_{2}, E_{2}\right)$ be n-vertex graphs with maximum degrees $\Delta\left(G_{i}\right)=\Delta_{i}$ for $i=1,2$. Let $2 \Delta_{1} \Delta_{2} \leq n$. $G_{1}$ and $G_{2}$ do not pack if and only if one of $G_{1}$ and $G_{2}$ is a perfect matching and the other either is $K_{\frac{n}{2}, \frac{n}{2}}$ with $\frac{n}{2}$ odd or contains $K_{\frac{n}{2}+1}$.

One can view Theorem 1.2 as a very small step towards the well-known conjecture by Bollobás and Eldridge [2] that any two $n$-vertex graphs $G_{1}$ and $G_{2}$ with maximum degrees $\Delta_{1}$ and $\Delta_{2}$ pack provided that $\left(\Delta_{1}+1\right)\left(\Delta_{2}+1\right) \leq n+1$. In the next section we give the outline of the proof and state all the lemmas. In the subsequent sections we prove the lemmas.

\section{Outline of the proof}

Say that a pair $\left(G_{1}, G_{2}\right)$ of graphs is a critical pair if $G_{1}$ and $G_{2}$ do not pack, but for each $e_{1} \in E\left(G_{1}\right), G_{1}-e_{1}$ and $G_{2}$ pack, and for each $e_{2} \in E\left(G_{2}\right), G_{1}$ and $G_{2}-e_{2}$ pack.

Consider 1-1 mappings of $V_{1}$ onto $V_{2}$. The result of each such mapping $f$ will be considered as the (multi)graph $G=G_{f}$ with labelled edges (with labels 1 and 2) whose vertices are pairs $(u, f(u))$ for $u \in V_{1}$, and for which two vertices $\left(u_{1}, f\left(u_{1}\right)\right)$ and $\left(u_{2}, f\left(u_{2}\right)\right)$ are connected by an edge in $E_{1}$ (respectively, $\left.E_{2}\right)$ if $u_{1} u_{2} \in E_{1}$ (respectively, $f\left(u_{1}\right) f\left(u_{2}\right) \in E_{2}$ ). We will use the expression $i$-neighbours of a vertex to denote its neighbours under $E_{i}$, $i=1,2$. For each such mapping $f$, define a $\left(u_{1}, u_{2} ; i, j\right)$-link to be a path of length two from $\left(u_{1}, f\left(u_{1}\right)\right)$ to $\left(u_{2}, f\left(u_{2}\right)\right)$ whose first edge has label $i$ and the second edge has label $j$, $i, j \in\{1,2\}$. For notational simplicity, we will often use $u$ instead of $(u, f(u))$. A $\left(u_{1}, u_{2}\right)$ switch means replacing the mapping $f$ with a mapping $f^{\prime}$ that differs from $f$ only in that $f^{\prime}\left(u_{1}\right)=f\left(u_{2}\right)$ and $f^{\prime}\left(u_{2}\right)=f\left(u_{1}\right)$. In a $\left(u_{1}, u_{2}\right)$-switch, all the 2-neighbours of $u_{1}$ become the 2-neighbours of $u_{2}$ and vice versa.

For $e \in E_{1}$ (respectively, $e \in E_{2}$ ), an e-packing of $G_{1}$ and $G_{2}$ is a mapping $f$ of $V_{1}$ onto $V_{2}$ such that $e$ is the only edge in $E_{1}$ (respectively, $E_{2}$ ) that shares its incident vertices with an edge from $E_{2}$ (respectively, $E_{1}$ ). Such a packing exists for every edge $e$ in a critical pair. In this case, we will say that $e$ is a conflicting edge. Each $e$-packing will be also called a quasi-packing if $e$ is not specified.

The proof of the following lemma essentially repeats the proof of Theorem 1.1 by Sauer and Spencer.

Lemma 2.1. Let $\left(G_{1}, G_{2}\right)$ be a critical pair and $2 \Delta_{1} \Delta_{2} \leq n$. Given any $e=u_{1} u_{1}^{\prime} \in E_{1}$, the following statements hold for any e-packing of $G_{1}$ and $G_{2}$.

(i) For every $u \neq u_{1}^{\prime}$, there exists either a unique $\left(u_{1}, u ; 1,2\right)$-link or a unique $\left(u_{1}, u ; 2,1\right)$-link.

(ii) There is neither a $\left(u_{1}, u_{1}^{\prime} ; 1,2\right)$-link nor a $\left(u_{1}, u_{1}^{\prime} ; 2,1\right)$-link.

(iii) $2 \operatorname{deg}_{G_{1}}\left(u_{1}\right) \operatorname{deg}_{G_{2}}\left(u_{1}\right)=n$.

Since $e$ was chosen arbitrarily and the roles of $G_{1}$ and $G_{2}$ are symmetric, we have, in particular, that $2 \Delta_{1} \Delta_{2}=n$ and $\operatorname{deg}\left(w_{i}\right) \in\left\{0, \Delta_{i}\right\}$ for all $w_{i} \in V_{i}, i=1,2$.

By this lemma, we have a packing of $G_{1}$ and $G_{2}$ if $2 \Delta_{1} \Delta_{2}<n$, and we need to consider below only the case when $2 \Delta_{1} \Delta_{2}=n$ and in particular, $n$ is even. The main part of our proof will be the following characterization of possibilities for $G_{1}$ and $G_{2}$. 
Lemma 2.2. If $2 \Delta_{1} \Delta_{2}=n$ and $\left(G_{1}, G_{2}\right)$ is a critical pair, then every component of $G_{i}$ is either $K_{\Delta_{i}, \Delta_{i}}$ with $\Delta_{i}$ odd, or an isolated vertex, or $K_{\Delta_{i}+1}, i=1,2$.

This lemma alone allows us to settle the case of $\min \left\{\Delta_{1}, \Delta_{2}\right\}=1$. Indeed, suppose $\Delta_{2}=1$, i.e., $G_{2}$ is a matching. Then $\Delta_{1}=\frac{n}{2}$. If $G_{1}$ contains $K_{\Delta_{1}, \Delta_{1}}$, then simply $G_{1}=K_{\frac{n}{2}, \frac{n}{2}}$. And the only case when we cannot pack $K_{\frac{n}{2}}, \frac{n}{2}$ with a matching is when the matching is perfect and $\frac{n}{2}$ is odd. If $G_{1} \neq K_{\frac{n}{2}, \frac{n}{2}}$, then by Lemma 2.2, it contains $K_{\frac{n}{2}+1}$, and in this case $G_{1}$ has no room for any other non-trivial components described by Lemma 2.2. Again, if the graph consisting of $K_{\frac{n}{2}+1}$ and $\frac{n}{2}-1$ isolated vertices does not pack with a matching, then the matching has to be perfect. This confirms the theorem for the case when $\Delta_{2}=1$ or $\Delta_{1}=1$.

The next lemma forbids some of the possibilities allowed by Lemma 2.2.

Lemma 2.3. Let $\Delta_{1}, \Delta_{2}>1$ and $2 \Delta_{1} \Delta_{2}=n$. If $\left(G_{1}, G_{2}\right)$ is a critical pair and the conflicted edge in a quasi-packing belongs to a component $H$ of $G_{2}$ isomorphic to $K_{\Delta_{2}, \Delta_{2}}$, then every component of $G_{1}$ sharing vertices with $H$ is $K_{\Delta_{1}, \Delta_{1}}$.

Since for every edge $e \in E\left(G_{1}\right) \cup E\left(G_{2}\right)$, there exists an $e$-packing, and each quasipacking of $G_{1}$ and $G_{2}$ is a connected graph, Lemma 2.3 yields that if at least one component of $G_{2}$ is $K_{\Delta_{2}, \Delta_{2}}$ or at least one component of $G_{1}$ is $K_{\Delta_{1}, \Delta_{1}}$, then every component of $G_{2}$ is $K_{\Delta_{2}, \Delta_{2}}$ and every component of $G_{1}$ is $K_{\Delta_{1}, \Delta_{1}}$. Furthermore, in this case both $\Delta_{1}$ and $\Delta_{2}$ are odd, $G_{1}$ consists of $\Delta_{2}$ copies of $K_{\Delta_{1}, \Delta_{1}}$ and $G_{1}$ consists of $\Delta_{1}$ copies of $K_{\Delta_{2}, \Delta_{2}}$. Thus the following two packing results will complete the proof of the theorem.

Lemma 2.4. Let $\Delta_{1}, \Delta_{2}>1$ and $2 \Delta_{1} \Delta_{2}=n$. If every non-trivial component of $G_{i}$ is $K_{\Delta_{i}+1}$, $i=1,2$, then $G_{1}$ and $G_{2}$ pack.

Proof. Let the components of $G_{1}$ be $H_{1}, \ldots, H_{k}, H_{k+1}, \ldots, H_{k+m}$, where each of $H_{1}, \ldots, H_{k}$ is a $K_{\Delta_{1}+1}$, and each of $H_{k+1}, \ldots, H_{k+m}$ is an isolated vertex. We may assume that $m \leq \Delta_{1}$ (otherwise, we may form additional copies of $K_{\Delta_{1}+1}$ from the isolated vertices without violating the conditions of the lemma). Thus

$$
k \geq \frac{n-\Delta_{1}}{\Delta_{1}+1}=\frac{2 \Delta_{1} \Delta_{2}-\Delta_{1}}{\Delta_{1}+1}=\Delta_{2}+\frac{\Delta_{1} \Delta_{2}-\Delta_{1}-\Delta_{2}}{\Delta_{1}+1}=\Delta_{2}+\frac{\left(\Delta_{1}-1\right)\left(\Delta_{2}-1\right)-1}{\left(\Delta_{1}+1\right)} .
$$

If $\Delta_{1} \geq 3$, or $\Delta_{2} \geq 3$, or $m<\Delta_{1}$, this implies that $k \geq \Delta_{2}+1$. In this case, order $H_{1}, \ldots, H_{k}$ cyclically and map each vertex of a copy of $K_{\Delta_{2}+1}$ in $G_{2}$ to a vertex in $H_{i}$ in this order. After all the vertices in $H_{i}, 1 \leq i \leq k$, are exhausted, continue on to the isolated vertices. Since $k \geq \Delta_{2}+1$, no two vertices in a component of $G_{2}$ will be assigned to the same component of $G_{1}$, giving a valid packing.

If $\Delta_{1}=\Delta_{2}=2$ and $m=\Delta_{1}=2$, then we need to pack two 8-vertex graphs each consisting of two triangles and two isolated vertices. Let $u_{1} u_{2} u_{3}$ and $v_{1} v_{2} v_{3}$ be the two triangles of $G_{1}$ and $x$ and $y$ be the isolated vertices in $G_{1}$. Map the vertices of the two triangles of $G_{2}$ to $u_{2} v_{2} x$ and $u_{3} v_{3} y$, respectively, and the isolated vertices to $u_{1}$ and $v_{1}$.

Lemma 2.5. Suppose that $\Delta_{1}, \Delta_{2} \geq 3$ and odd, and $2 \Delta_{1} \Delta_{2}=n$. If $G_{1}$ consists of $\Delta_{2}$ copies of $K_{\Delta_{1}, \Delta_{1}}$ and $G_{2}$ consists of $\Delta_{1}$ copies of $K_{\Delta_{2}, \Delta_{2}}$, then $G_{1}$ and $G_{2}$ pack. 
Proof. We may assume that $\Delta_{1} \geq \Delta_{2}$. Suppose that the components of $G_{1}$ are $H_{1}, \ldots, H_{\Delta_{2}}$ and the partite sets of $H_{i}$ are

$$
\left\{u_{(i-1) \Delta_{1}+1}, u_{(i-1) \Delta_{1}+2}, \ldots, u_{i \Delta_{1}}\right\} \quad \text { and } \quad\left\{u_{\frac{n}{2}+(i-1) \Delta_{1}+1}, u_{\frac{n}{2}+(i-1) \Delta_{1}+2}, \ldots, u_{\frac{n}{2}+i \Delta_{1}}\right\}
$$

for $i=1, \ldots, \Delta_{2}$. We map the first $2 \Delta_{2}$ vertices in this numeration to a copy of $K_{\Delta_{2}, \Delta_{2}}$ in $G_{2}$, then the second $2 \Delta_{2}$ vertices in this numeration to another copy of $K_{\Delta_{2}, \Delta_{2}}$ in $G_{2}$, and so on. In order to see that this is a packing, we will check that any $2 \Delta_{2}$ consecutive vertices in our numeration of $V\left(G_{1}\right)$ form an independent set. Indeed, consider the set $U_{j}=\left\{u_{j}, u_{j+1}, \ldots, u_{j+2 \Delta_{2}-1}\right\}$ for some $j$. Note that $u_{i}$ is adjacent in $G_{1}$ to $u_{j}$ only if $|i-j|>\frac{1}{2} n-\Delta_{1}$. But $\Delta_{2} \geq 3$, hence $\frac{1}{2} n-\Delta_{1} \geq 3 \Delta_{1}-\Delta_{1} \geq 2 \Delta_{2}$.

Thus, to complete the proof of Theorem 1.2 it is enough to prove Lemmas 2.1, 2.2, and 2.3.

\section{Proof of Lemmas 2.1 and 2.2}

Proof of Lemma 2.1. Let $\left(G_{1}, G_{2}\right)$ be a critical pair with $\Delta_{1} \Delta_{2} \leq \frac{n}{2}$. Given an $e_{1} \in E_{1}$, consider an $e_{1}$-packing of $\left(G_{1}, G_{2}\right)$ with $e_{1}=u_{1} u_{1}^{\prime}$.

Suppose that for some $u \neq u_{1}^{\prime}$ there exists neither a $\left(u_{1}, u ; 1,2\right)$-link nor a $\left(u_{1}, u ; 2,1\right)$-link. Then make a $\left(u_{1}, u\right)$-switch. Any new conflicting edge must be incident to either $u_{1}$ or $u$. If there is a conflicting edge incident to $u_{1}$ then, under the original quasi-packing, there is either a $\left(u, u_{1} ; 1,2\right)$-link or $u_{1} u$ is a conflicting edge. The latter does not happen, since $u \neq u_{1}^{\prime}$. Similarly, if there is a conflicting edge incident to $u$ then there is a $\left(u, u_{1} ; 2,1\right)$-link in the original quasi-packing. This contradicts our assumption, and hence there exists at least one $\left(u_{1}, u ; 1,2\right)$ - or $\left(u_{1}, u ; 2,1\right)$-link.

Since each of $d_{G_{1}}\left(u_{1}\right)$ 1-neighbours of $u_{1}$ (respectively, each of $d_{G_{2}}\left(f\left(u_{1}\right)\right)$ 2-neighbours of $u_{1}$ ) has at most $\Delta_{2}$ 2-neighbours (respectively, at most $\Delta_{1}$ 1-neighbours), at most $d_{G_{1}}\left(u_{1}\right) \Delta_{2}+d_{G_{2}}\left(f\left(u_{1}\right)\right) \Delta_{1}$ such links start at $u_{1}$. Two of these links also finish at $u_{1}$. We have already seen that each $u \neq u_{1}^{\prime}, u_{1}$, is connected by such a link to $u_{1}$. This gives

$$
n-2 \leq d_{G_{1}}\left(u_{1}\right) \Delta_{2}+d_{G_{2}}\left(f\left(u_{1}\right)\right) \Delta_{1}-2 .
$$

Under the conditions of the lemma, this is possible only if $d_{G_{1}}\left(u_{1}\right)=\Delta_{1}, d_{G_{2}}\left(f\left(u_{1}\right)\right)=\Delta_{2}$, $2 \Delta_{1} \Delta_{2}=n$, and no link was wasted, that is, no $u \neq u_{1}$ is connected with $u_{1}$ by two or more links, and $u_{1}^{\prime}$ is not connected by a link with $u_{1}$. This proves the lemma.

Now we will prove Lemma 2.2 in a series of claims. Let $\left(G_{1}, G_{2}\right)$ be a critical pair with $\Delta_{1} \Delta_{2}=\frac{n}{2}$. Suppose that $G_{1}$ is not the union of copies of $K_{\Delta_{1}+1}$ and isolated vertices. Let $H_{1}$ be a component of $G_{1}$ which is neither $K_{\Delta_{1}+1}$ nor an isolated vertex. By Lemma 2.1, $H_{1}$ is $\Delta_{1}$-regular. Thus, $H_{1}$ contains an induced $P_{3}=\left(u_{1}^{\prime}, u_{1}, u_{2}\right)$. Let $e_{1}=u_{1} u_{1}^{\prime}$. Consider an $e_{1}$-packing $f$ of $G_{1}$ and $G_{2}$.

Claim 3.1. If $u_{1} u_{2} \in E_{1}$ and $u_{2} u_{1}^{\prime} \notin E_{1}$, then there exists $a\left(u_{1}, u_{2} ; 1,2\right)$-link.

Proof. By Lemma 2.1, there exists either a unique $\left(u_{1}, u_{2} ; 1,2\right)$-link or a unique $\left(u_{1}, u_{2} ; 2,1\right)$ link. If the claim does not hold, then there exists a $\left(u_{1}, u_{2} ; 2,1\right)$-link. Make a $\left(u_{1}^{\prime}, u_{2}\right)$-switch. 

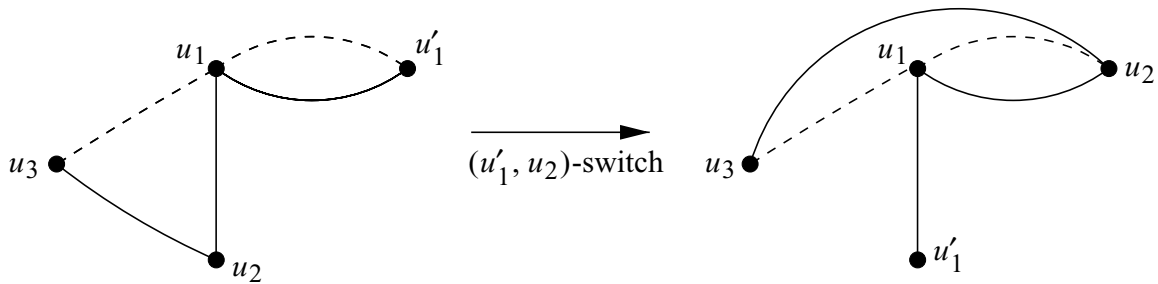

Figure 1. Claim 3.1 with edges in $E_{1}$ denoted by - and edges in $E_{2}$ denoted by - - - .

Then there exists a $\left(u_{1}, u_{2} ; 2,1\right)$-link under the new mapping $f^{\prime}$ which is a $u_{1} u_{2}$-packing. This contradicts Lemma 2.1(ii). See Figure 1.

Claim 3.2. Under the same conditions, let $u_{3}$ be the intermediate vertex on the $\left(u_{1}, u_{2} ; 1,2\right)$ link. Then $u_{3} u_{1}^{\prime} \notin E_{1}$ and $u_{3} u_{1}^{\prime} \notin E_{2}$.

Proof. Otherwise, there exist two $\left(u_{1}, u_{3} ; i, j\right)$-links, $i \neq j, i, j \in\{1,2\}, u_{1} \rightarrow u_{2} \rightarrow u_{3}$ and $u_{1} \rightarrow u_{1}^{\prime} \rightarrow u_{3}$, contradicting Lemma 2.1(i).

Claim 3.3. Under the conditions of Claim 3.2,

(i) $u_{2}$ is the only common neighbour of $u_{1}$ and $u_{3}$,

(ii) there is no common 1-neighbour of $u_{1}$ and $u_{1}^{\prime}$,

(iii) all 1-neighbours of $u_{2}$ are also 1-neighbours of $u_{1}^{\prime}$.

Proof. (i) Let $u_{4}$ be another common neighbour of $u_{1}$ and $u_{3}$. By Claim 3.2, $u_{4} \neq u_{1}^{\prime}$. By Lemma 2.1 , there is either a $\left(u_{1}, u_{3} ; 1,1\right)$-link or a $\left(u_{1}, u_{3} ; 2,2\right)$-link through $u_{4}$.

If there is a $\left(u_{1}, u_{3} ; 1,1\right)$-link through $u_{4}$, then make a $\left(u_{1}^{\prime}, u_{4}\right)$-switch. Then there exist two $\left(u_{1}, u_{3} ; i, j\right)$-links, $u_{1} \rightarrow u_{2} \rightarrow u_{3}$ and $u_{1} \rightarrow u_{4} \rightarrow u_{3}$, under the new mapping $f^{\prime}$, which is a $u_{1} u_{4}$-packing, $i \neq j, i, j \in\{1,2\}$. This contradicts Lemma 2.1(i). See Figure 2(a).

If there is a $\left(u_{1}, u_{3} ; 2,2\right)$-link through $u_{4}$, then make a $\left(u_{1}, u_{1}^{\prime}\right)$-switch. Then there exist two $\left(u_{1}, u_{4} ; 1,2\right)$-links under the new mapping $f^{\prime}$, which is a $u_{1} u_{1}^{\prime}$-packing. This contradicts Lemma 2.1(i). See Figure 2(b).

(ii) Let $u_{5}$ be a common 1-neighbour of $u_{1}$ and $u_{1}^{\prime}$. Make a $\left(u_{1}^{\prime}, u_{3}\right)$-switch. By (i), there is no edge between $u_{1}^{\prime}$ and $u_{3}$, and hence none exists after the switch as well. In the new mapping $f^{\prime}$, a $u_{1} u_{3}$-packing with $u_{1}^{\prime}$ as the intermediate vertex on the $\left(u_{1}, u_{2} ; 1,2\right)$-link, $u_{5}$ is a second common neighbour of $u_{1}$ and $u_{1}^{\prime}$, contradicting (i). See Figure 3.

(iii) Suppose $u_{2}^{\prime}$ is a 1-neighbour of $u_{2}$ but not of $u_{1}^{\prime}$. By Lemma 2.1(i), there exists a unique $\left(u_{1}, u_{2}^{\prime} ; i, j\right)$-link whose intermediate vertex $u$ is not $u_{1}^{\prime}$, since $u_{2}^{\prime}$ is not a 1 -neighbour of $u_{1}^{\prime}$ and $u_{2}^{\prime}$ is also not a 2-neighbour of $u_{1}^{\prime}$, otherwise there would be two $\left(u_{1}^{\prime}, u_{2} ; 2,1\right)$-links (through $u_{1}$ and $u_{2}^{\prime}$ ).

If $u=u_{3}$, then $u_{3}$ is a 2-neighbour of $u_{2}^{\prime}$. The $\left(u_{1}, u_{2}\right)$-switch leads to two $\left(u_{3}, u_{2} ; 2,1\right)$-links under the new mapping $f^{\prime}$, which is a $u_{1} u_{3}$-packing. This contradicts Lemma 2.1(i). 


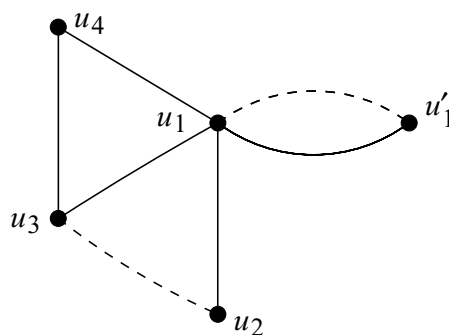

$\left(u_{1}^{\prime}, u_{4}\right)$-switch

(a)
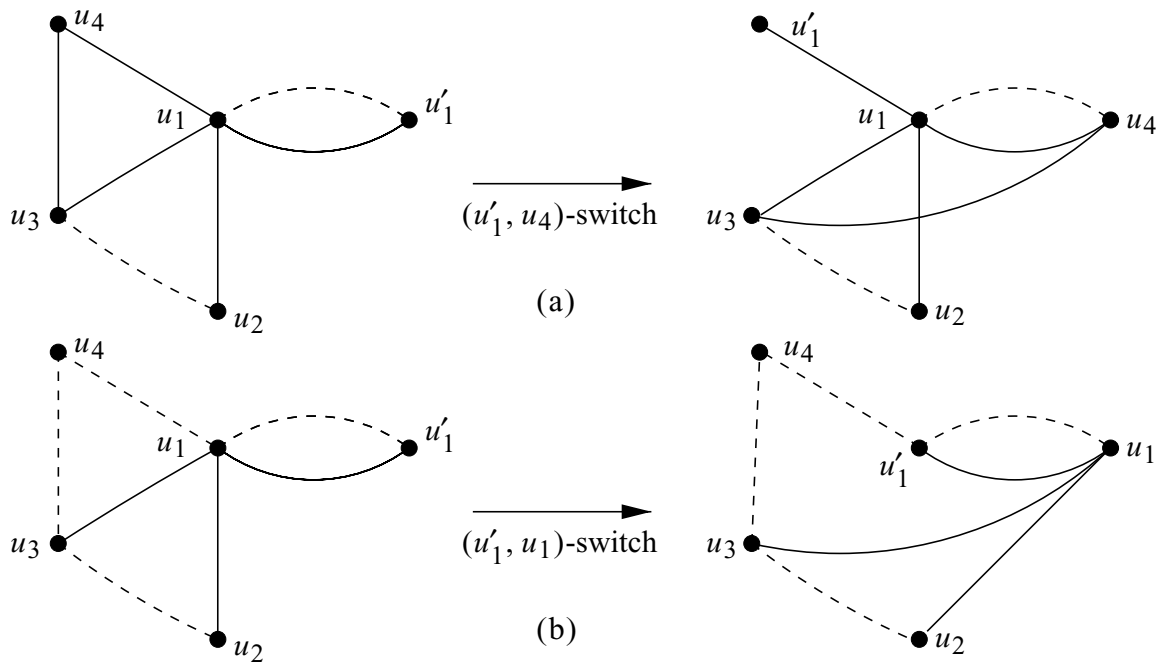

$\left(u_{1}^{\prime}, u_{1}\right)$-switch

(b)

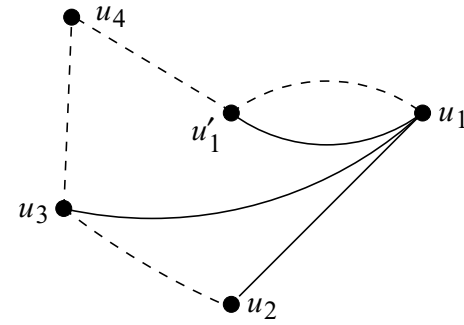

Figure 2. Claim 3.3(i).
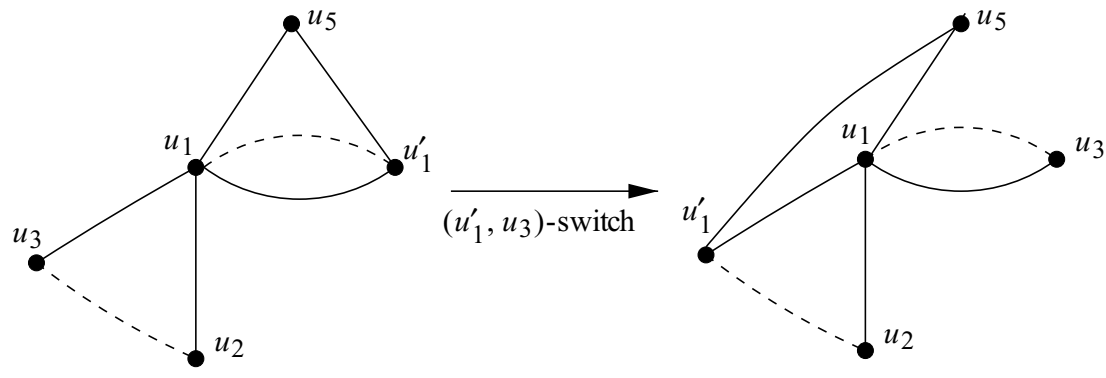

Figure 3. Claim 3.3(ii).
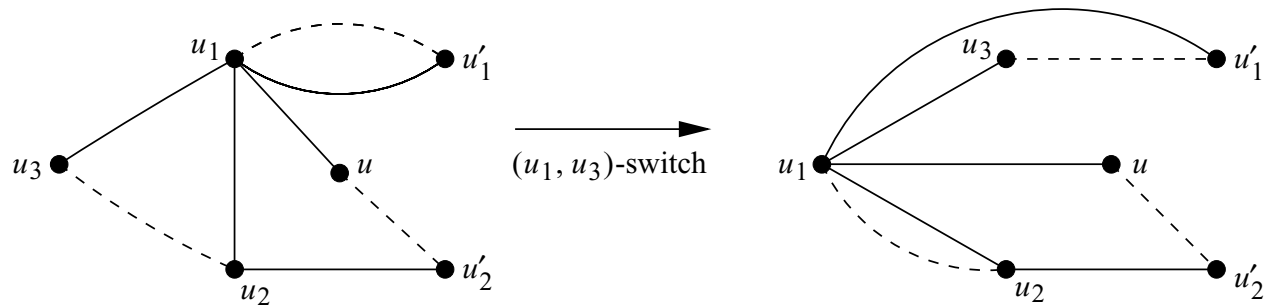

Figure 4. Claim 3.3(iii).

If $u \neq u_{3}$ then there exists a $\left(u_{1}, u_{2}^{\prime} ; i, j\right)$-link through intermediate vertex $u, i \neq j, i, j \in$ $\{1,2\}$. The $\left(u_{1}, u_{3}\right)$-switch leads to two $\left(u_{1}, u_{2}^{\prime} ; i, j\right)$-links under the new mapping $f^{\prime}$, a $u_{1} u_{2}$-packing. This contradicts Lemma 2.1(i). See Figure 4. 
As noted above, each vertex in the non-trivial component $H_{1}$ of $G_{1}$ has $\Delta_{1}$ 1-neighbours. If $u_{2}^{\prime}\left(\neq u_{2}\right)$, a 1 -neighbour of $u_{1}$, has a 1-neighbour that is not a 1-neighbour of $u_{2}$ then by Claim 3.3 with $u_{2}^{\prime}$ playing the role of $u_{2}, u_{1}^{\prime}$ will have more than $\Delta_{1}$ 1-neighbours, a contradiction. Hence, all the 1-neighbours of $u_{1}$ have exactly the same $\Delta_{1} 1$-neighbours, that is, the component of $G_{1}$ containing $u_{1}$ is $K_{\Delta_{1}, \Delta_{1}}$. Also, by Claim 3.3(i), there is a pairing of 1-neighbours of $u_{1}$ (respectively, $u_{1}^{\prime}$ ) other than $u_{1}^{\prime}$ (respectively, $u_{1}$ ), with a 2-edge between each such pair and no other 2-edges induced by the vertices of $H_{1}$. This means that these 2-edges induce a matching. By counting the 1-neighbours of $u_{1}$, we see that $\Delta_{1}$ is odd, as $u_{1}^{\prime}$ is also a 1-neighbour of $u_{1}$. This completes the proof of Lemma 2.2.

\section{Proof of Lemma 2.3}

Suppose that $e_{0}=u_{1,1} u_{2,1}$ is the only conflicting edge in a quasi-packing of $G_{1}$ with $G_{2}$ and that the component $H_{2}$ of $G_{2}$ containing $e_{0}$ is a $K_{\Delta_{2}, \Delta_{2}}$ with partite sets $U_{1}=\left\{u_{1,1}, u_{1,2}, \ldots, u_{1, \Delta_{2}}\right\}$ and $U_{2}=\left\{u_{2,1}, u_{2,2}, \ldots, u_{2, \Delta_{2}}\right\}$. Let $H_{1}$ be the component of $G_{1}$ containing $e_{0}$.

Claim 4.1. $V\left(H_{1}\right) \cap V\left(H_{2}\right)=\left\{u_{1,1}, u_{2,1}\right\}$.

Proof. If a vertex $u \in V\left(H_{2}\right)-\left\{u_{1,1}, u_{2,1}\right\}$ belongs to $V\left(H_{1}\right)$, then it is adjacent in $G_{1}$ to $\left\{u_{1,1}, u_{2,1}\right\}$. Suppose, for definiteness, that it is adjacent to $u_{1,1}$. Since $u u_{1,1}$ is not a conflicting edge, $u \in U_{1}$. But then there is a $\left(u_{1,1}, u_{2,1} ; 1,2\right)$-link, a contradiction to Lemma 2.1.

Claim 4.2. Every component of $G_{1}$ shares with $\mathrm{H}_{2}$ either 0 or 2 vertices.

Proof. Let $u \in U_{1}-u_{1,1}$. By Lemma 2.1 , there is either a $\left(u_{2,1}, u ; 1,2\right)$-link or a $\left(u_{2,1}, u ; 2,1\right)$ link. The former cannot happen by Claim 4.1, so $u$ should be adjacent in $G_{1}$ to another vertex in $U_{1}$. Moreover, if it were adjacent in $G_{1}$ to more than one vertex in $U_{1}$, then there would be more than one $\left(u_{2,1}, u ; 2,1\right)$-link, a contradiction to Lemma 2.1. Thus, each vertex of $H_{2}$ is adjacent in $G_{1}$ to exactly one other vertex of $H_{1}$.

Consider now any component $H_{0}$ of $G_{1}$ sharing a vertex with $H_{2}$ and distinct from $H_{1}$. By Claim 4.2, it shares with $\mathrm{H}_{2}$ exactly two vertices (from the same partite set). Suppose for definiteness that $V\left(H_{0}\right) \cap V\left(H_{2}\right)=\left\{u_{1,2}, u_{1,3}\right\}$. If $H_{0}=K_{\Delta_{1}+1}$, then, since $\Delta_{1} \geq 2$, the graph $H_{0}$ contains a vertex $u_{0}$ adjacent to both $u_{1,2}$ and $u_{1,3}$. But then there are at least two $\left(u_{2,1}, u_{0} ; 2,1\right)$-links, a contradiction to Lemma 2.1. Thus, each component of $G_{1}$ sharing a vertex with $H_{2}$ and distinct from $H_{1}$ is a $K_{\Delta_{1}, \Delta_{1}}$.

Consider a $\left(u_{2,1}, u_{1,2}\right)$-switch. By Lemma 2.1, the new mapping $f^{\prime}$ is also a quasi-mapping. Furthermore, now $H_{1}$ does not have conflicting edges with $H_{2}$. Thus, by the previous paragraph, $H_{1}$ is a $K_{\Delta_{1}, \Delta_{1}}$. This finishes the proof of Lemma 2.3.

\section{Acknowledgements}

The authors thank the referee for his/her helpful comments that led to an improved manuscript. 


\section{References}

[1] Bollobás, B. (1978) Extremal Graph Theory, Academic Press, London/New York.

[2] Bollobás, B. and Eldridge, S. E. (1978) Packing of graphs and applications to computational complexity. J. Combin. Theory Ser. B 25 105-124.

[3] Catlin, P. A. (1974) Subgraphs of graphs I. Discrete Math. 10 225-233.

[4] Sauer, N. and Spencer, J. (1978) Edge disjoint placement of graphs. J. Combin. Theory Ser. B 25 295-302.

[5] Wozniak, M. (1997) Packing of graphs. Dissertationes Math. 36278 pp.

[6] Yap, H. P. (1988) Packing of graphs: A survey. Discrete Math. 72 395-404. 